\title{
Performance Analysis of Fast Charging Stations for G2V and V2G Micro-grid Systems
}

\author{
Veerpratap Meena, Dr Vinay Pant, Arunendra Verma, Kanchan Chariya
}

\begin{abstract}
In order to promote the switching from ICE vehicles to more environment friendly EVs, a network of fast charging stations is necessary. A lot of research is being conducted in this area in order to design efficient models of chargers along with developing new technologies to mitigate the current problems encountered such as the charging time, incapability to charge multiple vehicles at a time etc. This paper presents the different ways of classifying $\mathrm{EV}$ battery charging technologies and also a topological survey of different charging stations proposed in the literature. A three-phase bidirectional charger and a modular fast charger proposed in the literature are also presented along with their modelling, control strategies and simulation results to analyse their respective performances.
\end{abstract}

Keywords:- bidirectional topology, fast charging, Vehicle to Grid (V2G), grid support, charging station topology, modularity.

\section{INTRODUCTION}

In recent years electric vehicles (EVs) have started to become attractive alternatives to the conventional internal combustion engine (ICE) vehicles. Their advantages include energy efficiency, fuel and maintenance cost savings, reduced emissions and their benefits towards public health, the ability to provide ancillary services, the modification of the demand curve and the V2G application. On the other hand, the use of these systems also has several issues, such us the higher price of the vehicle, the need of widespread fast charging infrastructure and the overload of the power networks.

EVs can be charged in residential areas from homes or from public outlets. There are basically 4 levels of charging on the basis of charging time. Level 1 is the slow charging of EVs from home outlets. Level 2 also includes slow charging but from public charging stations. Level 3 charging refers to fast charging from special three phase charging stations while [8] level 4 charging refers to DC fast charging.

V2G technology refers to the power flow from vehicle to grid. This application when effectively introduced in a smart grid can improve the efficiency, reliability and stability of the supply grid. It can also p rovide ancillary services and assist in demand side management strategies.

This paper mainly focuses on different fast charging topologies in which the G2V application can also be incorporated. There are a number of desired features for fast charging stations [2]. A bidirectional topology is essential to incorporate grid support services in the charging station. The charging stations should have a provision for integration of renewable energy sources (RES) and Battery energy storages (BES). These help in reducing the overload on the supply grid due to multiple vehicles charging at a time. Further the fast charging stations should have high power density. Power density is the ratio of the power available to the area of the charging station. The charging stations should also have lesser number of components and simple control strategies in order to ensure reliable operations.

\section{CLASSIFICATION OF BATTERY CHARGING TECHNOLOGIES}

Conductive and inductive chargers: Battery charging technologies can be classified as conductive and inductive on the basis of the interfacing between the grid and the vehicle. A Conductive charger connects the charging station outlet to the connector of the EV using a cable, manually plugged in by the driver. Alternatively, the power is transferred magnetically in the case of an inductive charger. This type of charger comprises two parts: the grid interface where the AC current is take in, rectified and converted to a high frequency; and the secondary side, installed inside the vehicle, which consists of an AC/DC converter connected to the battery. At the terminal of each side, a winding is placed forming a transformer with the help of which the power transfer is carried out. The relative ratings of these chargers on various aspects are compared in Figure 1(a) [1].

$>$ On-Board and Off-Board Chargers: Chargers can be termed as On-Board chargers if a part of the charger's structure is included in the vehicle and if not, they are called Off-Board chargers. On-Board charging topologies are limited to level 1 and 2 (slow charging) because of the weight, cost and space constraints but the vehicles can be charged anywhere if a compatible power source is available. Off board chargers a are usually used for fast charging purposes from charging stations. Figure 1(b). compares the ratings of the two types of chargers.

Bidirectional and unidirectional chargers: A battery charger is an appliance composed of power electronic circuits that are employed in the conversion of electrical energy. These power converters are differentiated based on the direction of the power exchange between the grid and the battery. The power flow through a charging structure can be unidirectional $(\mathrm{G} 2 \mathrm{~V}$ mode) or 
bidirectional ( $\mathrm{G} 2 \mathrm{~V}$ and $\mathrm{V} 2 \mathrm{G}$ modes), as depicted in Figure 2. The bidirectional charger even though more complicated, can prove beneficial to the grid.

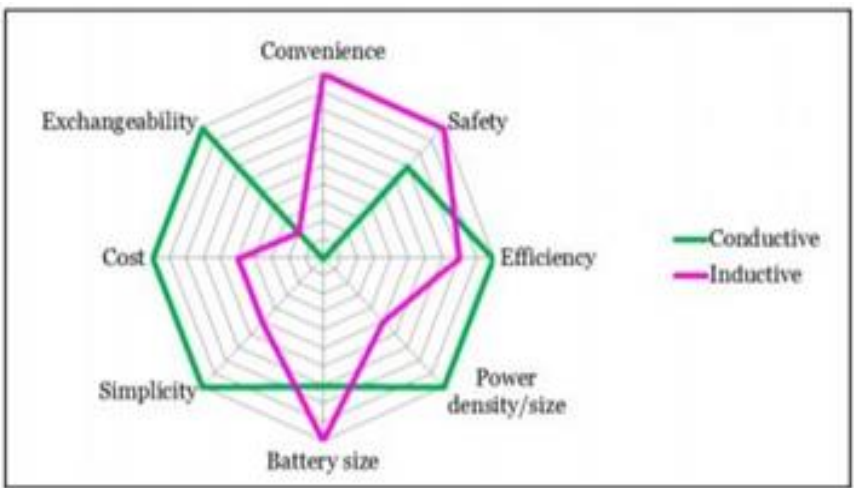

(a)

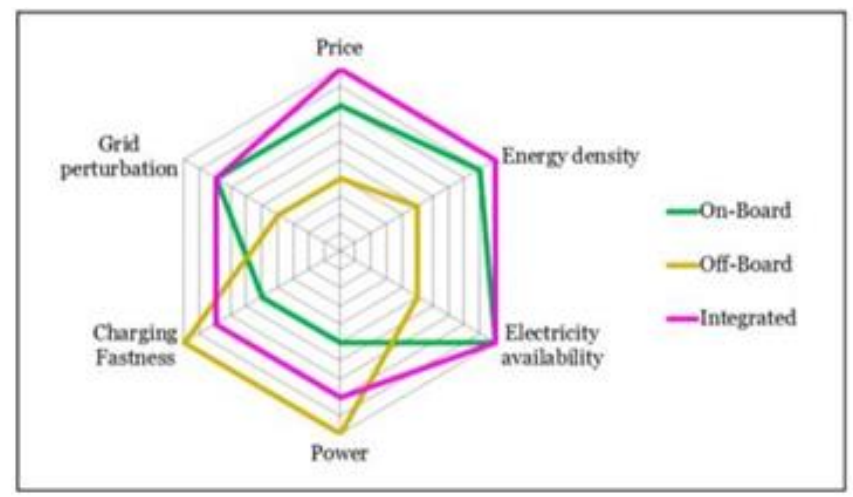

(b)

Fig 1:- Comparison between (a) Conduvtive and Inductive chargers (b) On-Board, Off-Board and Integrated chargers.

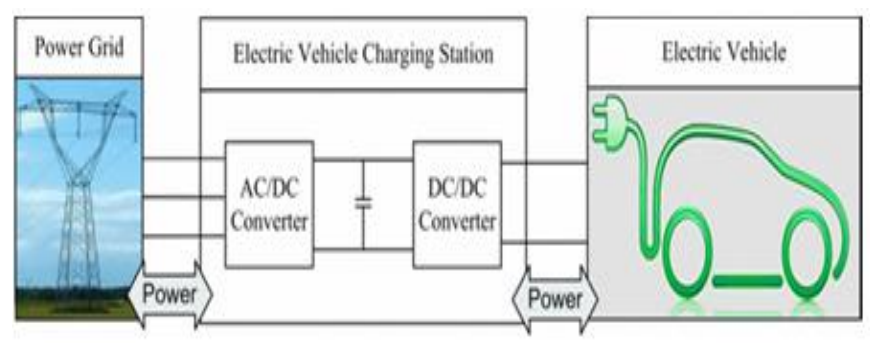

Fig 2:- Power flow in a general charger's structure

High voltage and low voltage batteries: Depending on the voltage level of the Battery packs used in the electric vehicles, the topologies of the chargers may vary. For high voltage batteries only an AC/DC converter is required while for batteries with lower voltage ratings a DC/DC converter is also utilized in order to step down the voltage to a value that is compatible with the battery. The presented topologies employ MOSFETs for low power, IGBTs for medium power and GTOs for high power applications. [1]

\section{CHARGING STATION TOPOLOGIES}

Several charging stations topologies have been proposed in the literature. They can be grouped under the following three categories[2]. An example for each topology is also presented.

\section{A. Topologies having back to back AC/DC/DC converters}

This category includes an AC/DC converter which is interfaced to the grid using a distribution transformer and a filter. A dc-link is used to feed the DC/DC converters connected to it. The dc-link makes it possible to have high modularity as several applications such as storage systems and renewable energy sources integration can be served.

\section{$>$ Bi-directional charging stations}

This topology helps in reducing the negative impacts of fast charging on the supply grid by incorporating RESs and BESs. It also allows the easy implementation of G2V and V2G operations. In [3] a $1.05 \mathrm{kVA} 10$-slot charging station having bidirectional converters is modelled. This charging station was found to perform well under a charging load of $900 \mathrm{~kW}$. It was interfaced with a strong medium voltage grid and so the impact of fast charging on the grid was not studied. The proposed model is shown in Figure 3.

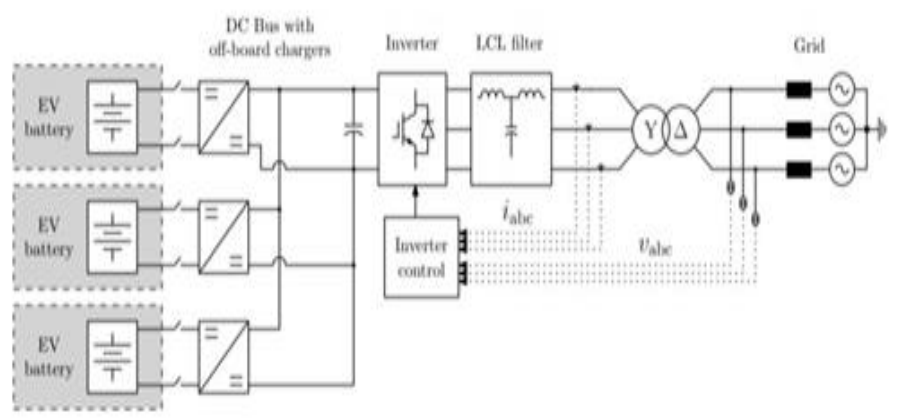

Fig 3:- Bidirectional charging station with back to back AC/DC/DC converters

\section{Unidirectional Charging stations}

A unidirectional design of the above discussed topology is shown in Figure 4 [2]. The DC link voltage control and the active filtering of the AC current harmonics produced by the initial 12-pulse diode rectifier is carried out by two buck-boost converters. This model is not feasible to be fed from weaker points in the distribution grid as they cannot provide grid support and hence voltage drop compensation. 


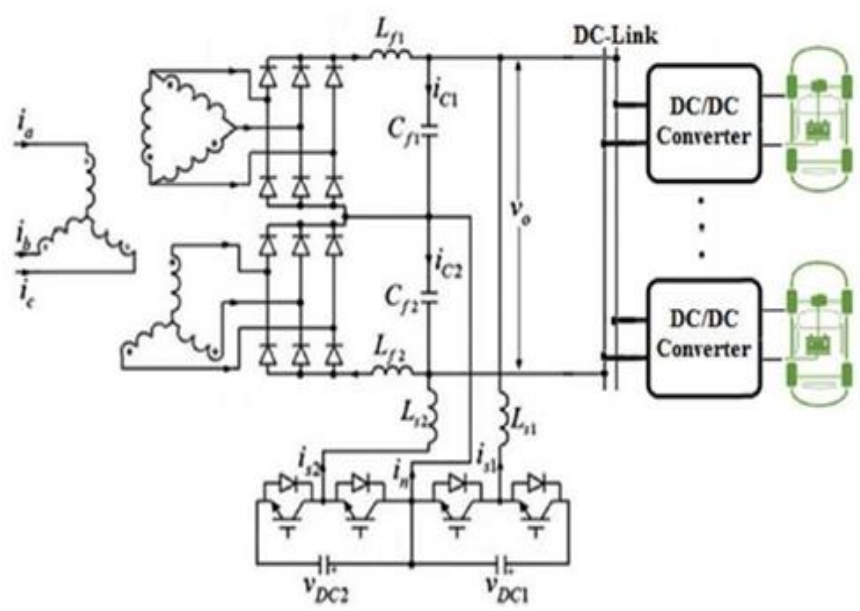

Fig 4:- Unidirectional charging station with bach to back AC/DC/DC converters.

\section{B. Multiport stations sharing common AC link}

In [4] a multiterminal charging facility which is also fit for bidirectional operation is proposed. Each EV charger is connected to the grid through a unity ratio low frequency transformer as an isolating device. This is followed by an AC $(50 \mathrm{~Hz}) / \mathrm{DC} / \mathrm{AC}$ (high frequency) conversion. The obtained high frequency $\mathrm{AC}$ voltage is transferred to the charger through a coreless coils. The structure of the proposed topology is shown in Figure 5. In this case also the charging station is incapable of providing voltage support or power factor improvement to the grid. Also, this charging station was not modelled for fast charging of EVs. The increased count of components and control circuits leads to the reduction of the reliability and efficiency levels.

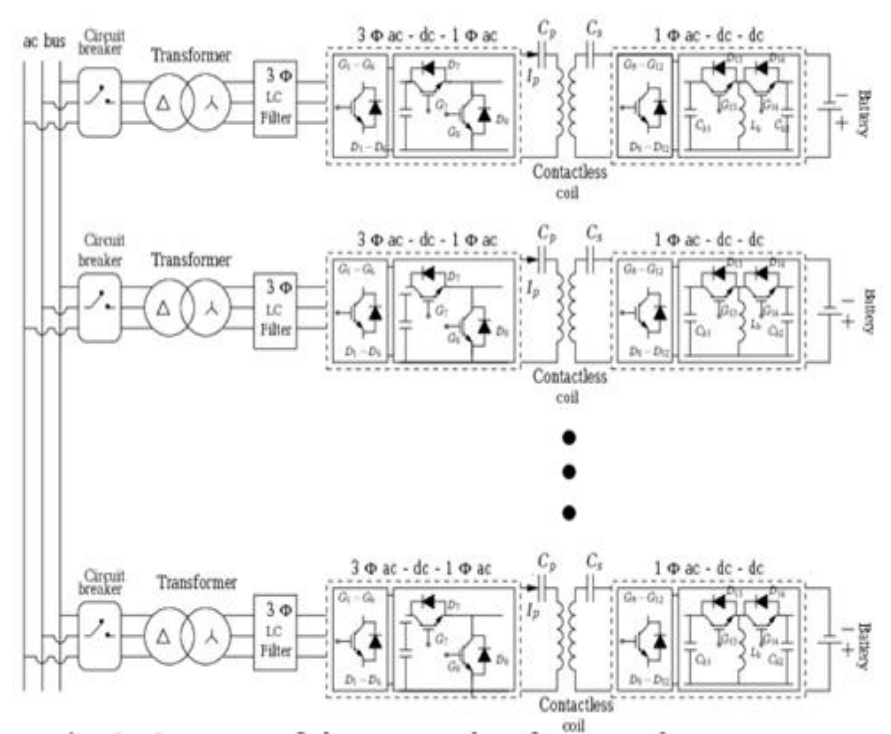

\section{Transformerless charging stations}

In order to improve the power density of the charging stations some models have been proposed by removing the low frequency transformer from the topology. This requires the charging station to be fed from either a low voltage grid or directly from a medium voltage grid. When fed from low voltage grids the losses are high due to increased current rates. The proposed models have used medium voltage cascaded multi-level inverters to feed the charger directly from a medium voltage grid $(4.8 \mathrm{kV})$. Each phase of the grid to charger interface comprises of several AC/DC/DC converters forming a multilevel converter. Moreover, a battery storage is used at the dc end in place of a capacitor bank. The structure of a similar topology proposed in [5] is shown in Figure 6.

The different topologies can be compared on the basis of the desired attributes of a fast charging station. Table 1 summarizes the features of the different topologies.

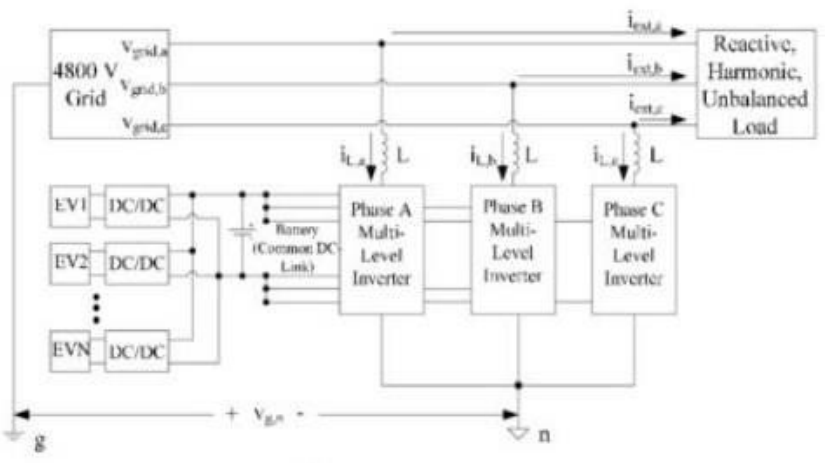

(a)

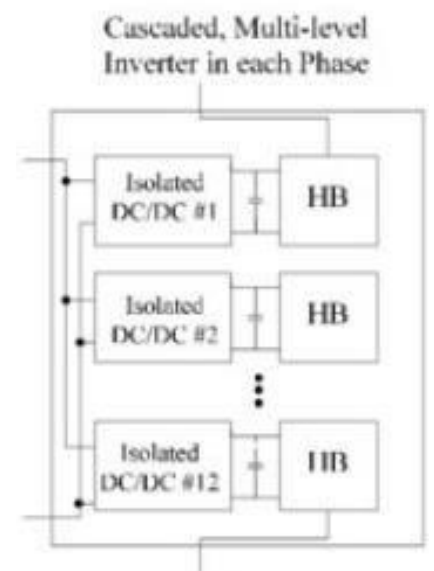

(b)

Fig 6:- Charging station having cascaded multilevel converters.

Fig 5:- Multiport charging station.

\begin{tabular}{|c|c|c|c|c|c|c|c|}
\hline Type & Bi- directional & Grid support & RES integration & BES integration & Power density & Component count & Control complexity \\
\hline A(1) & Yes & All* & Yes & Yes & Medium & Low & Low \\
\hline A(2) & No & APF & No & Yes & Medium & Low & Low \\
\hline B & Yes & V2G & No & No & Low & High & Low \\
\hline C & Yes & All* & Yes & Yes & High & High & High \\
\hline
\end{tabular}

*-S2G, V2G, Active power filter (APF) 


\section{MODELLING AND SIMULATION OF G2V AND V2G SYSTEMS}

In this section two charging station topologies are taken up and their electrical circuit and control strategies are presented. The results of simulation of these models are also included.

\section{A. Electrical Model}

The first charging station is a bidirectional charging station with an AC/DC converter and a DC/DC converter [6]. The electrical model is as shown in Figure 7.

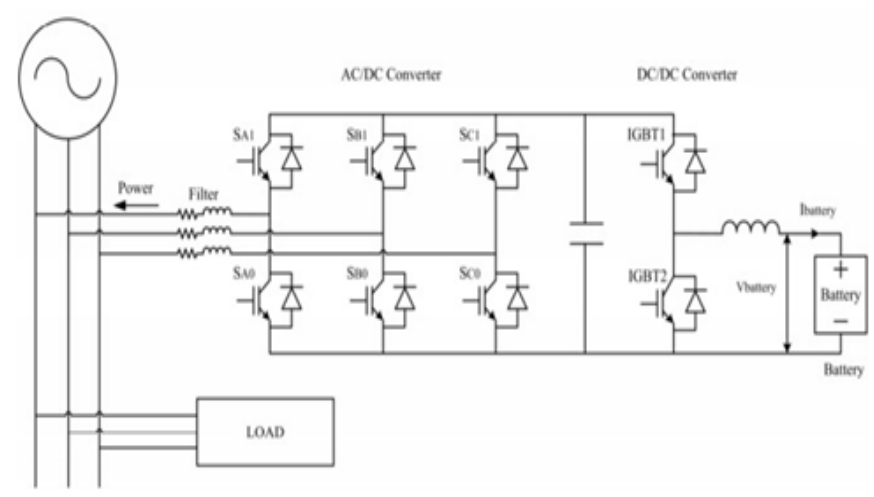

Fig 7:- Electrical model of bidirectional charger

The second topology which can be said as an example of a modular fast charging station. It employs a combination of three single phase modules in place of the single set of three phase converters [7]. This is to increase the modularity of the system and guarantee low voltage operation. The electrical model is shown in Figure 8.

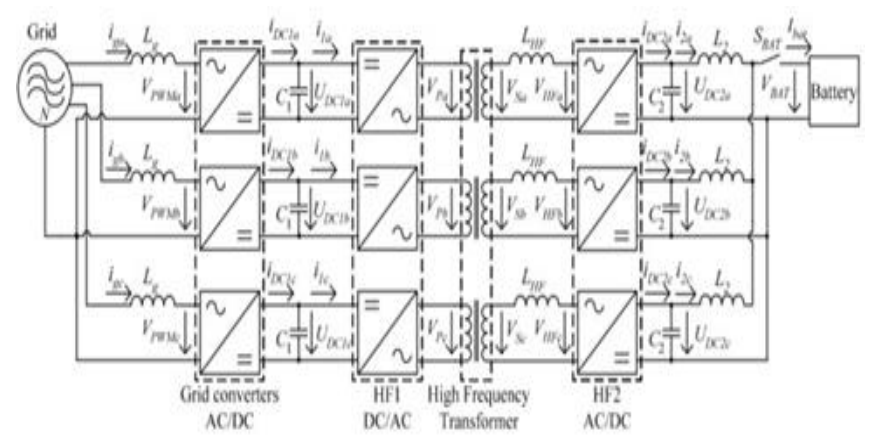

Fig 8:- Electrical model of modular fast charger.

\section{B. Control Strategy}

The first model uses a direct control technique for controlling the bidirectional power flow through the charging mechanism. The control is achieved by operating the DC/DC converter in the desired mode. Buck mode operation requires IGBT1 to be triggered whereas Boost mode operation is obtained by triggering IGBT2. The control strategy of this model is explained in Figure 9. Initially the controller of the DC/DC converter compares the reference value and measured value of the battery current. The error is passed through a PI controller for tuning. The output of the PI controller is then sent for sinusoidal pulse width modulation (SPWM) to generate pulses. The pulses generated are directed to IGBT1 for charging and IGBT2 for discharging operations respectively. The IGBT which is not triggered is kept off during. both the modes of operation.
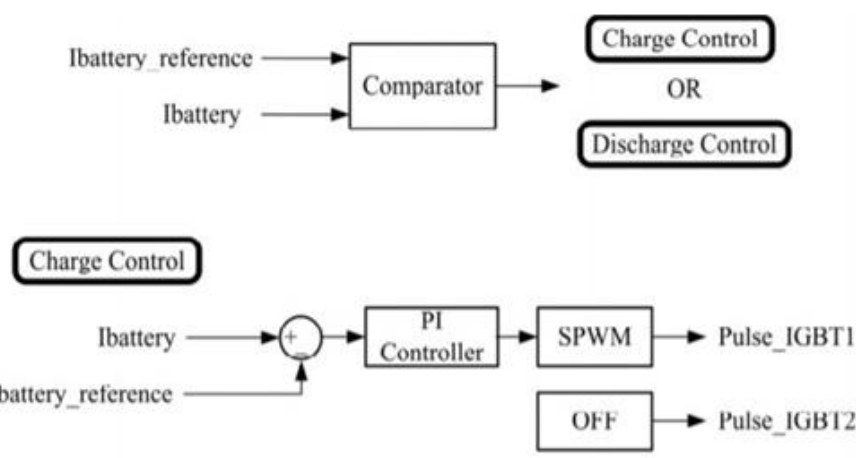

Discharge Control

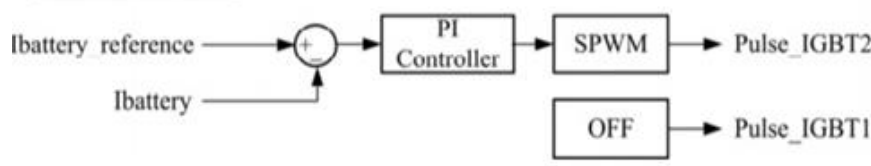

Fig 9:- Control strategy of proposed bidirectional converter.

In the second charging system also PI controllers are employed for the controllers excluding the battery current controller for which an integral compensator is used. The control of the grid current, DC link voltage, battery charging current and voltage are achieved by controlling appropriately the current through the grid side and battery side converters and the voltage across the respective DC capacitors. The block diagrams for the respective controllers are shown in Figure 10. In each case $\alpha$ represents the corresponding sensor's gain. The regulation of the power transfer to and from the battery is carried out by varying the displacement angle $\delta$ between the fundamental component of the voltage Vs and the voltage VHF as can be seen in Figure 10 (c).

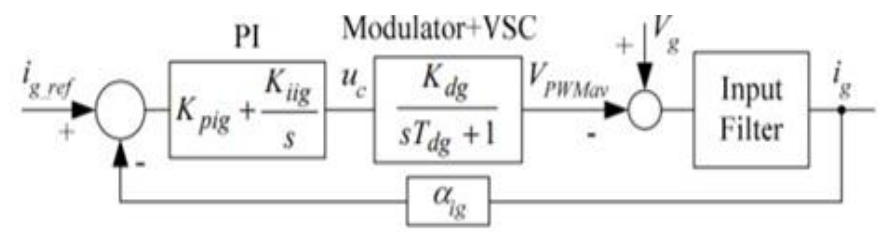

(a)

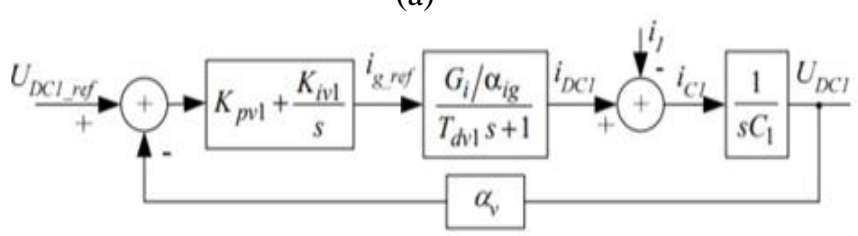

(b)

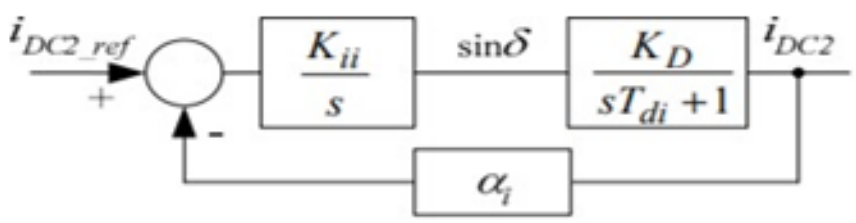

(c) 


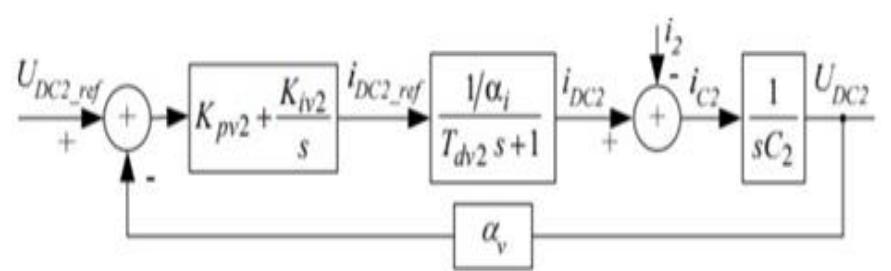

(d)

Fig 10:- (a) Grid current controller. (b) DC link voltage controller. (c) Battery current controller. (d) Battery side capacitor voltage controller

\section{Results of simulation}

The proposed models were simulated and their parameters were measured in order to analyse their performances. The State of charge (SOC) percentages of the batteries, battery voltages and currents of both topologies were plotted. They are as shown in Figures 11,12 and 13. The first model was tested under the operating modes namely fast charging, slow charging, fast discharging and slow discharging. The corresponding efficiencies of the charger for each mode of operation are listed in table 2. For the second topology also the test results are presented for $\mathrm{G} 2 \mathrm{~V}$ and $\mathrm{V} 2 \mathrm{G}$ modes. Also, the grid current and voltages for the second model are found to be almost in phase. This implies efficient power factor correction of the charger.

On analyzing the graphs, the second model is found to have faster charging rates. The SOC in this case was found to increase from $20-80 \%$ in less than 30 minutes.

On the other hand, in the second case the SOC starting from $50 \%$ showed an increase of $0.5 \%$ in 5 minutes.
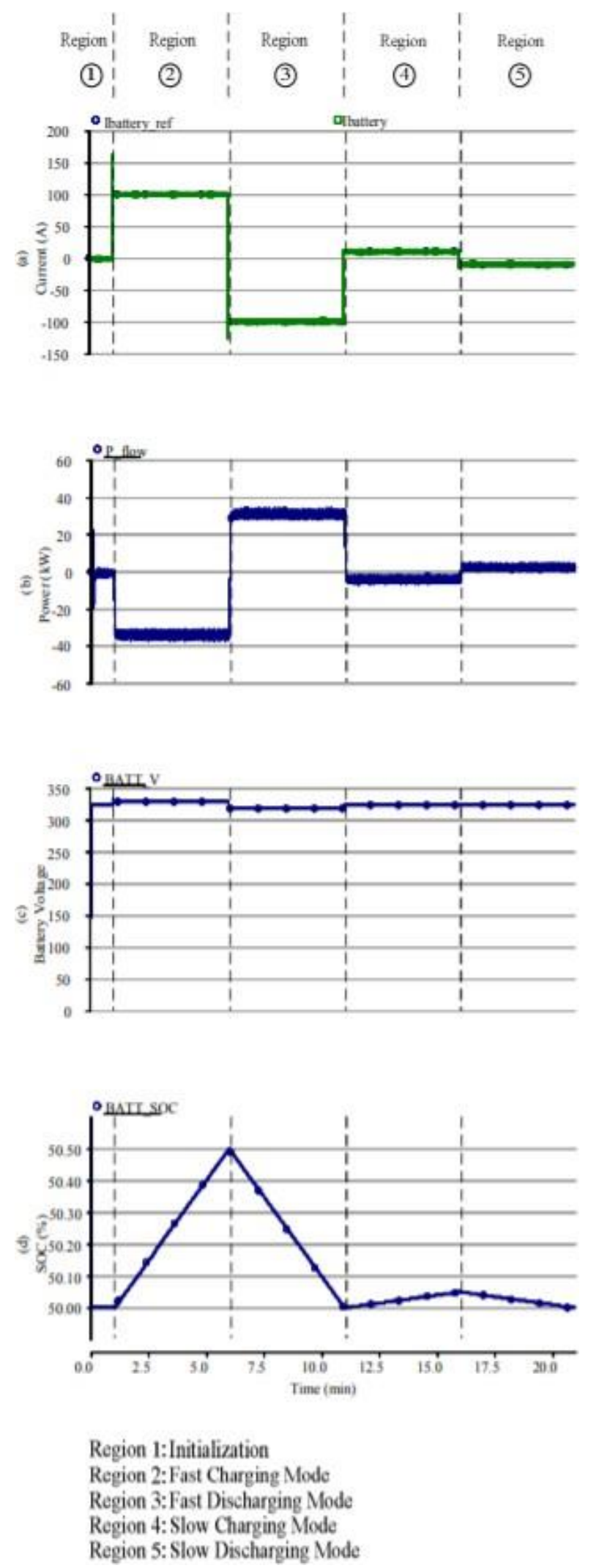

Fig 11:- Simulation results of first charger model (a) Battery current. (b) Battery Power. (c) Battery Voltage. (d) Batter SOC. 


\begin{tabular}{|c|c|c|c|c|c|c|}
\hline \multirow{2}{*}{$\begin{array}{c}\text { Operating } \\
\text { M ode }\end{array}$} & \multicolumn{3}{|c|}{ Charging } & \multicolumn{3}{c|}{ Discharging } \\
\cline { 2 - 7 } & Pin (kW) & Pout (kW) & Efficiency (\%) & Pin (kW) & Pout (kW) & Efficiency (\%) \\
\hline Slow mode & 3.30 & 3.25 & 98.48 & 3.20 & 3.15 & 98.43 \\
\hline Fast mode & 33.50 & 32.50 & 97.01 & 31.50 & 30.80 & 97.77 \\
\hline
\end{tabular}

Table 2:- Efficiencies of different operating modes.
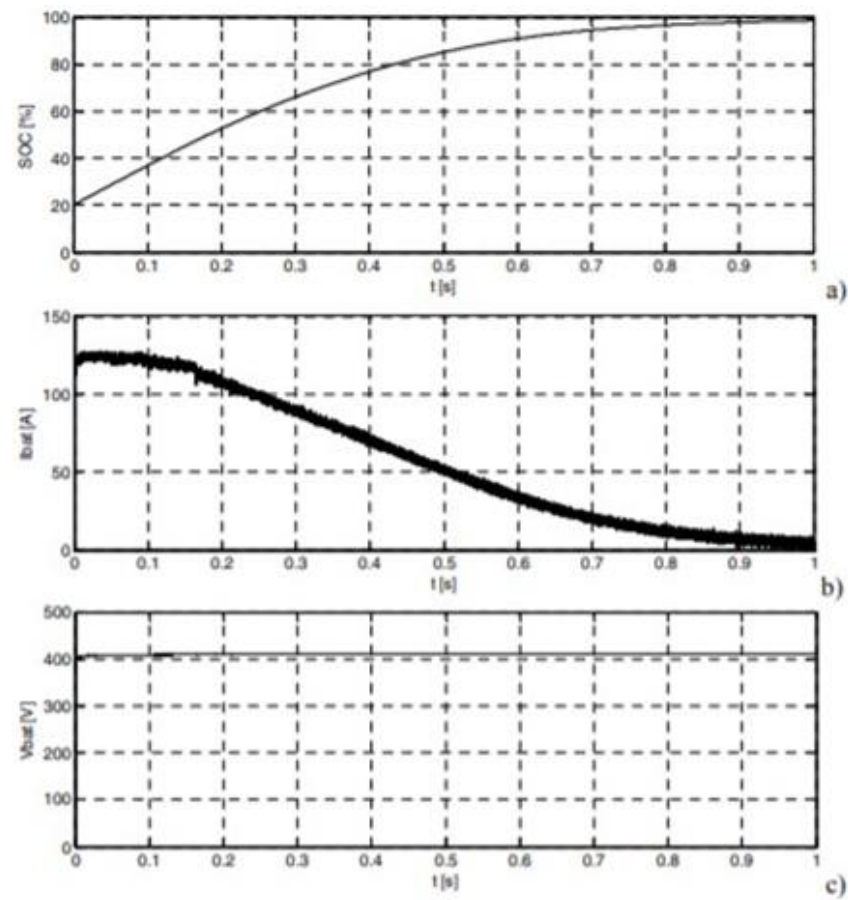

Fig 12:- Simulation results of second charger model for G2V operation. (a) SOC. (b) Battery current. (c) Battery voltage.
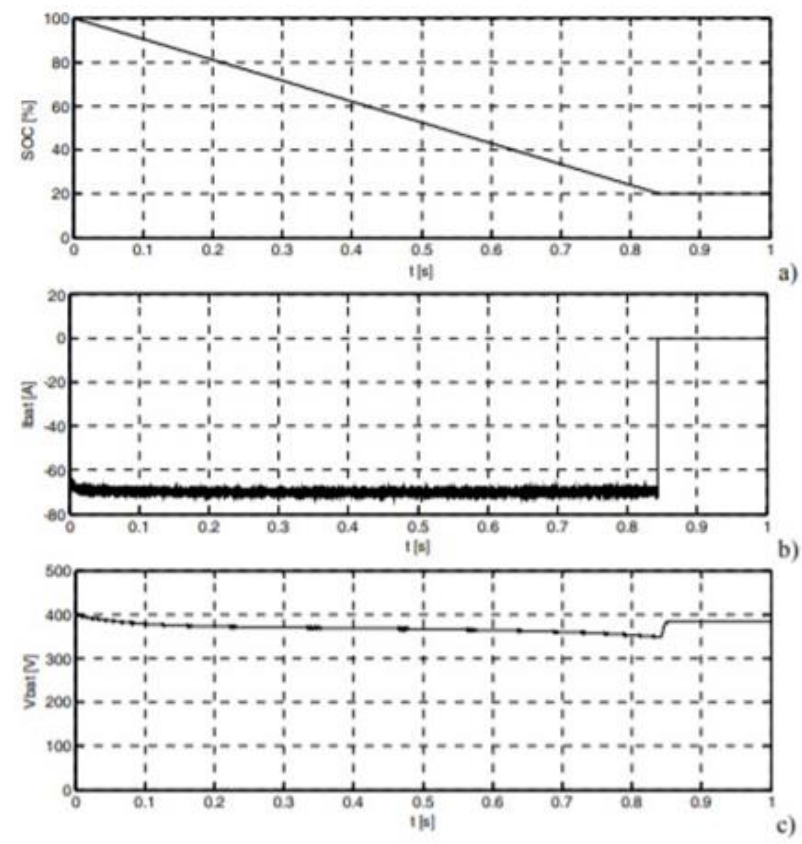

Fig 13:- Simulation results of second charger model for V2G operation. (a) SOC. (b) Battery current. (c) Battery voltage.

\section{CONCLUSION}

The widespread implementation of reliable and efficient fast charging stations for electric vehicles with bidirectional power transfer capabilities, modularity and high power density are required for the uptake of the EV technology which in turn will prove beneficial economically as well as environmentally. In this paper several topologies were briefly discussed and the modelling and simulation results of two models were compared and their performances analysed. The former one being a threephase bidirectional fast charger with back to back AC/DC/DC chargers and the latter being a modular fast charger. The efficiencies were found to meet the desired limits for both G2V and V2G modes of operation. The modular charger provided a provision for charging multiple vehicles at a time along with slightly faster rates of charging compared to the first model.

\section{REFERENCES}

[1]. María Garcés Quílez, Mohamed Abdel-Monem, Mohamed El Baghdadi, Yang Yang, Joeri Van MierloID and Omar Hegazy, "Modelling, Analysis and Performance Evaluation of Power Conversion Unit in G2V/V2G Application-A Review," Energies, 11, 1082, April 2018.

[2]. Mohsen Ahmadi, N. Mithulananthan and Rahul Sharma, "A Review on Topologies for Fast Charging Stations for Electric Vehicles," IEEE International Conference on Power System Technology, July 2016.

[3]. Arancibia, Arnaldo, and Kai Strunz. "Modeling of an electric vehicle charging station for fast DC charging." IEEE International Electric Vehicle Conference(IEVC), 2012.

[4]. Joy, T. P., Kannan Thirugnanam, and Pranaw Kumar. "A multi-point Bidirectional Contactless Charging System in a charging station suitable for EVs and PHEVs applications." Annual IEEE India Conference (INDICON), 2013.

[5]. Crosier, Russell, Shuo Wang, and Mohamed Jamshidi. "A 4800-V gridconnected electric vehicle charging station that provides STACOM-APF functions with a bi-directional, multi-level, cascaded converter." Twenty-Seventh Annual IEEE Applied Power Electronics Conference and Exposition (APEC), 2012.

[6]. Kang Miao Tan, Vigna K. Ramachandaramurthy and Jia Ying Yong, "Bidirectional Battery Charger for Electric Vehicle," IEEE Innovative Smart Grid Technologies - Asia (ISGT ASIA), 2014. 
[7]. Ricardo G. Gago, Sónia F. Pinto and José F. Silva, "G2V and V2G Electric Vehicle Charger for Smart Grids," IEEE International Smart Cities Conference, 2016

[8]. Kawther Fahem, Dhia Elhak Chariag and Lassaâd Sbita, "On-board Bidirectional Battery Chargers Topologies for Plug-in Hybrid Electric Vehicles," IEEE Internationa Conference on Green energy conversion systems, 2017.

[9]. Nitin Trivedi, Nikhil S. Gujar, Subrata Sarkar and S.P.S. Pundir, "Different fast charging methods and topologies for EV charging," IEEMA Engineer Infinite Conference (eTechNxT), 2018

[10]. T. Jalakas, I. Roasto and D.Vinnikov, "Analysis of Battery Charger Topologies for an Electric Vehicle," 2012 13th Biennial Baltic Electronics Conference, October 2012 